\title{
Langmuir
}

pubs.acs.org/Langmuir

(C) 2010 American Chemical Society

\section{Multivalent Interaction of Cyclodextrin Vesicles, Carbohydrate Guests, and Lectins: A Kinetic Investigation ${ }^{\dagger}$}

\author{
Raquel V. Vico, ${ }^{\star}$ Jens Voskuhl, ${ }^{\S}$ and Bart Jan Ravoo*, \\ *Instituto de Investigaciones en Fisicoquímica de Córdoba (INFIQC-UNC-CONICET), Departamento de \\ Química Orgánica, Facultad de Ciencias Químicas, Universidad Nacional de Córdoba, Haya de la Torre y \\ Medina Allende, Ciudad Universitaria, X5000HUA Córdoba, Argentina, and ${ }^{\S}$ Organic Chemistry Institute, \\ Westfälische Wilhelms-Universität Münster, Corrensstrasse 40, 48149 Münster, Germany
}

Received September 28, 2010. Revised Manuscript Received November 8, 2010

\begin{abstract}
An artificial glycocalix self-assembles when unilamellar bilayer vesicles of amphiphilic $\beta$-cyclodextrins are decorated with maltose- and lactose-adamantane conjugates by host-guest interactions. The maltose-decorated vesicles aggregate in the presence of lectin concanavalin A whereas the lactose-decorated vesicles aggregate in the presence of lectin peanut agglutinin. The kinetics of the orthogonal multivalent interfacial interactions present in this ternary system of vesicles, carbohydrates, and lectins were studied by time-dependent measurements of the optical density at $400 \mathrm{~nm}$. The average vesicle and vesicle aggregate sizes were monitored by dynamic light scattering. The aggregation process was evaluated as a function of lectin concentration, vesicle concentration, and surface coverage of the vesicles by the carbohydrate-adamantane conjugates. The initial rate of vesicle aggregation scales linearly with the lectin as well as the cyclodextrin vesicle concentration. Furthermore, each lectin requires a characteristic critical density of carbohydrates at the vesicle surface. These observations allow a prediction of the response of the ternary supramolecular system at different concentrations of its components. Also, the effective binding site separation in a multivalent receptor such as a multiple binding site protein can be accurately determined. This methodology can be extended to multivalent noncovalent interactions in other ligand-receptor systems at interfaces.
\end{abstract}

\section{Introduction}

The extracellular surfaces of prokaryotic and eukaryotic cells display a dense layer of complex carbohydrates, which is commonly known as a "glycocalix". Carbohydrates in the glycocalix act as ligands for proteins in cell-cell and cell-matrix interactions. For example, the human blood types (A, B, AB, and O) originate from the presence of different oligosaccharides at the erythrocyte surface. Proteins that bind to carbohydrates in the glycocalix are generally called lectins. ${ }^{1}$ The association constant for a $1: 1$ carbohydrate-lectin complex is $K_{\mathrm{a}} \approx 10^{3}-10^{4} \mathrm{M}^{-1}$. Many lectins are homo-oligomer assemblies consisting of two to four identical subunits, each with a recognition site for a single carbohydrate. The multivalent interactions of lectins and carbohydrates mediate the recognition and adhesion of cells as well as the activation of cellular signaling. ${ }^{1}$ Also, the binding of antibodies to foreign cells and viruses and the infection of cells with virus are essentially mediated by protein-carbohydrate interactions at the cell surface. ${ }^{1}$

Liposomes and vesicles are versatile membrane mimetic systems. In particular, the dynamic multivalent molecular recognition of their outer membrane surface has become an increasingly versatile topic in supramolecular chemistry in recent years. ${ }^{2}$

\footnotetext{
${ }^{\dagger}$ Part of the Supramolecular Chemistry at Interfaces special issue.

*Corresponding author. Fax.: (+49)251-8336557. E-mail: b.j.ravoo@ uni-muenster.de.

(1) Lis, H.; Sharon, N. Chem. Rev. 1998, 98, 637-674.

(2) Voskuhl, J.; Ravoo, B. J. Chem. Soc. Rev. 2009, 38, 495-505.

(3) Curatolo, W.; Yau, A. O.; Small, D. M.; Sears, B. Biochemistry 1978, 17, 5740-5744.

(4) Sundler, R. FEBS Lett. 1982, 141, 11-13.

(5) (a) Kitano, H.; Sodha, K.; Kosaka, A. Bioconjugate Chem. 1995, 6, 131-134. (b) Tagawa, K.; Sendai, N.; Ohno, K.; Kawaguchi, T.; Kitano, H.; Matsunaga, T. Bioconjugate Chem. 1999, 10, 354-360. (c) Kitano, H.; Sumi, Y.; Tagawa, K. Bioconjugate Chem. 2001, 12, 56-61. (d) Kitano, H.; Ishino, Y.; Yabe, K. Langmuir 2001, 17, 2312-2316.
}

The interaction of natural glycolipids as well as synthetic membranebound carbohydrates ("neoglycolipids") with various lectins has been studied in detail by several groups. ${ }^{3-15}$ Because lectins possess multiple carbohydrate binding sites, the interaction of lectins with glycolipid and neoglycolipid vesicles results in the aggregation (or agglutination) of the vesicles, which can be detected in real time as an increase in the optical density of the vesicle solution. In cell biology, this type of experiment is known as an agglutination assay.

However, vesicles can also be decorated with functional molecules through noncovalent interaction. ${ }^{2}$ We have shown that

(6) Luzardo, M. D.; Lanio, M. E.; Alvarez, C.; Pazos, I. F.; Figueroa, S.; Verez, V.; Disalvo, E. A. Colloids Surf., B 2002, 26, 281-289.

(7) (a) Faivre, V.; Costa, M. D. L.; Boullanger, P.; Baszkin, A.; Rosilio, V Chem. Phys. Lipids 2003, 125, 147-159. (b) Guo, C. X.; Boullanger, P.; Liu, T.; Jiang, L. J. Phys. Chem. B 2005, 109, 18765-18771. (c) Ballut, S.; Makky, A.; Loock, B.; Michel, J. P.; Maillard, P.; Rosilio, V. Chem. Commun. 2009, 224-226. (d) Makky, A.; Michel, J. P.; Kasselouri, A.; Briand, E.; Maillard, P.; Rosilio, V. Langmuir 2010, 26, 12761-12768.

(8) Bondurant, B.; Last, J. A.; Waggoner, T. A.; Slade, A.; Sasaki, D. Y. Langmuir 2003, 19, 1829-1837.

(9) (a) Mazzaglia, A.; Forde, D.; Garozzo, D.; Malvagna, P.; Ravoo, B. J.; Darcy, R. Org. Biomol. Chem. 2004, 957-960. (b) Mazzaglia, A.; Valerio, A.; Villari, V.; Rencurosi, A.; Lay, L.; Spadaro, S.; Scolaro, L. M.; Micali, N. New J. Chem. 2006, 30, 1662-1668. (c) McNicholas, S.; Rencurosi, A.; Lay, L.; Mazzaglia, A.; Sturiale, L.; Perez, M.; Darcy, R. Biomacromolecules 2007, 8, 1851-1857.

(10) Lee, H.-K.; Park, K. M.; Jeon, Y. J.; Kim, D.; Oh, D. H.; Kim, H. S.; Park, C. K.; Kim, K. J. Am. Chem. Soc. 2005, 127, 5006-5007.

(11) Bandaru, N. M.; Sampath, S.; Jayaraman, N. Langmuir 2010, 21, 95919596.

(12) Tamiaki, H.; Azefu, Y.; Shibata, R.; Sato, R.; Toma, K. Colloids Surf., B 2006, 53, 87-93.

(13) Hassane, F. S.; Frisch, B.; Schuber, F. Bioconjugate Chem. 2006, 17, 849-854.

(14) (a) Park, J.; Rader, L. H. Thomas, G. B.; Danoff, E. J.; English, D. S DeShong, P. Soft Matter 2008, 4, 1916-1921. (b) Thomas, G. B.; Rader, L. H.; Park, J.; Abezgauz, L.; Danino, D.; DeShong, P.; English, D. S. J. Am. Chem. Soc. 2009, 131, 5471-5477.

(15) Noble, G. T.; Flitsch, S. L.; Liem, K. P.; Webb, S. J. Org. Biomol. Chem. 2009, 7, 5245-5254. 
Chart 1. Molecular Structures of Amphiphilic $\beta$-Cyclodextrin 1 (CD 1), Bifunctional Maltose-Adamantane Conjugate 2, and Bifunctional Lactose-Adamantane Conjugate 3
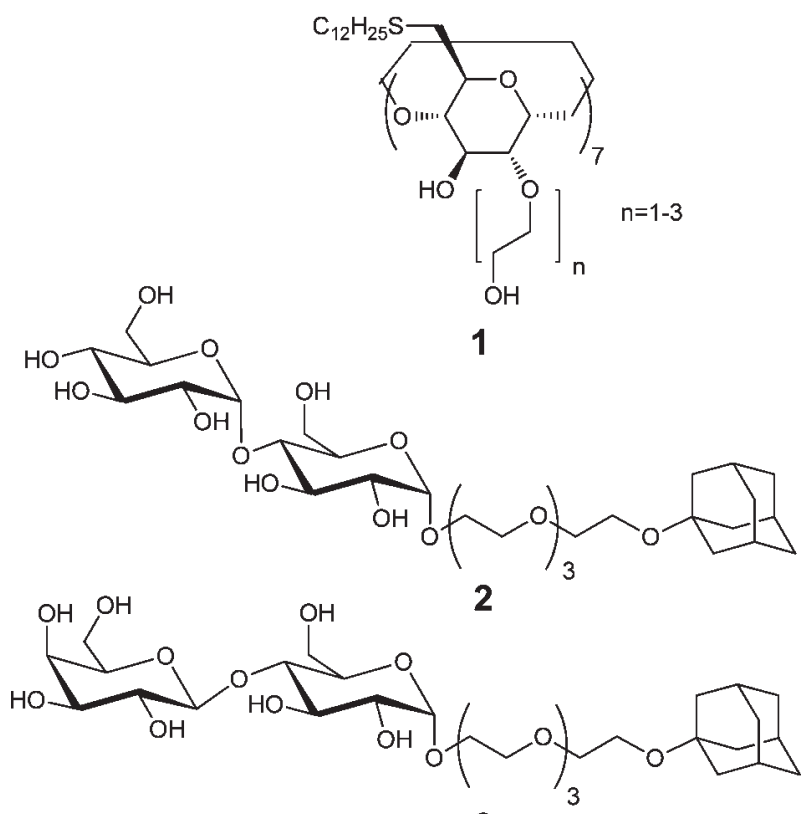

3

cyclodextrins modified with long hydrophobic alkyl tails and short hydrophilic oligo(ethylene glycol) headgroups form vesicles in aqueous solution. ${ }^{16}$ Amphiphilic cyclodextrins form unilamellar vesicles consisting of bilayers of cyclodextrins in which the hydrophobic tails are directed inward and the hydrophilic macrocycle headgroups are facing water, enclosing an aqueous interior. The cyclodextrin cavities at the surfaces of these vesicles are available for the complexation of hydrophobic guests such as adamantyl and $t$-butylbenzoyl derivatives. ${ }^{17}$ It was found that the host-guest interaction can be enhanced by multivalency in the case of guest polymers ${ }^{18}$ or guest molecules that associate at the vesicle surface through orthogonal noncovalent interactions such as metal-ligand coordination ${ }^{19}$ or hydrogen bonding. ${ }^{20,21}$ Furthermore, orthogonal multivalent interactions at the cyclodextrin vesicle surface can result in receptor clustering, ${ }^{22}$ vesicle clustering, ${ }^{19,21}$ and vesicle-nanotube transitions. ${ }^{20}$

In this work, we investigate the multivalent lectin-carbohydrate interaction of unilamellar bilayer vesicles of amphiphilic $\beta$-cyclodextrin 1 (CD 1) decorated with an artificial glycocalix composed of maltose $\mathbf{2}$ and/or lactose $\mathbf{3}$ (Chart 1). We have previously shown that an artificial glycocalix self-assembles via hostguest interactions of host vesicles of CD 1 with carbohydrate guests $\mathbf{2}$ and $\mathbf{3}$ (Scheme 1). ${ }^{23}$ Both carbohydrate-adamantane conjugates bind to $\beta$-cyclodextrin in a 1:1 complex $\left(K_{\mathrm{a}} \approx 4 \times 10^{4} \mathrm{M}^{-1}\right){ }^{23}$

(16) Ravoo, B. J.; Darcy, R. Angew. Chem., Int. Ed. 2000, 39, 4324-4326.

(17) Falvey, P.; Lim, C. W.; Darcy, R.; Revermann, T.; Karst, U.; Giesbers, M.; Marcelis, A. T. M.; Lazar, A.; Coleman, A. W.; Reinhoudt, D. N.; Ravoo, B. J. Chem.-Eur. J. 2005, 11, 1171-1180.

(18) Ravoo, B. J.; Jacquier, J. C.; Wenz, G. Angew. Chem., Int. Ed. 2003, 42, 2066-2070.

(19) Lim, C. W.; Crespo-Biel, O.; Stuart, M. C. A.; Reinhoudt, D. N.; Huskens, J.; Ravoo, B. J. Proc. Natl. Acad. Sci. U.S.A. 2007, 104, 6986-6991.

(20) Versluis, F.; Tomatsu, I.; Seda, K.; Fregonese, C.; Tepper, A. W. J. W.; Stuart, M. C. A.; Ravoo, B. J.; Koning, R. I.; Kros, A. J. Am. Chem. Soc. 2009, 131, 13186-13187.

(21) Voskuhl, J.; Fenske, T.; Stuart, M. C. A.; Wibbeling, B.; Schmuck, C.; Ravoo, B. J. Chem.-Eur. J. 2010, 16, 8300-8306.

(22) Lim, C. W.; Ravoo, B. J.; Reinhoudt, D. N. Chem. Commun. 2005, 56275629.

(23) Voskuhl, J.; Stuart, M. C. A.; Ravoo, B. J. Chem.-Eur. J. 2010, 16, 27902796.
The maltose-decorated vesicles aggregate (agglutinate) in the presence of lectin concanavalin A (ConA) whereas the lactosedecorated vesicles aggregate in the presence of lectin peanut agglutinin (PNA). ${ }^{23}$ In the self-assembled glycocalix, three carbohydrates are involved in orthogonal noncovalent interactions: CD $\mathbf{1}$ is a host for adamantane, maltose $\mathbf{2}$ is a ligand for ConA, and lactose $\mathbf{3}$ is a ligand for PNA.

The aim of this work is to elucidate the kinetics of the orthogonal multivalent interfacial interactions present in ternary systems of host vesicles, guest carbohydrates, and lectins and to establish in which way these interactions depend on the composition of the system (i.e., the concentration of the individual constituents). To this end, we performed a systematic kinetic study of the aggregation of cyclodextrin vesicles (CDV) decorated with carbohydrate guests in the presence of lectins ConA and PNA. Both ConA and PNA are tetramers at neutral $\mathrm{pH}$, and each lectin has four identical carbohydrate binding sites. ConA binds exclusively to $\alpha$-glucosides, $\alpha$-mannosides, and their derivatives (including maltose) and shows no significant affinity toward other carbohydrates (such as lactose). On the other hand, PNA binds exclusively to $\beta$-galactosides (including lactose) and shows no affinity toward other carbohydrates (such as maltose). The aggregation of CDV was monitored by using optical density and dynamic light scattering measurements. In the first part of this article, the aggregation of maltose-decorated CDV by ConA is investigated. In the second part, the aggregation of lactosedecorated CDV by PNA is investigated.

\section{Materials and Methods}

Materials. All chemicals and lectins used in this study were purchased from Acros Organics (Schwerte, Germany) or SigmaAldrich Chemie (Taufkirchen, Germany) and were used without further purification. Amphiphilic $\beta$-cyclodextrin 1, maltoseadamantane conjugate $\mathbf{2}$, and lactose-adamantane conjugate $\mathbf{3}$ were prepared as described. ${ }^{17,23}$

Methods. Unilamellar $\beta$-cyclodextrin vesicles (CDV) with an average diameter of $100-140 \mathrm{~nm}$ were obtained by the extrusion of a buffer solution with multilamellar vesicles through a $100 \mathrm{~nm}$ polycarbonate membrane in a Liposofast manual extruder. The concentration of CDV in the solutions was estimated from the concentration and molecular area of CD $1\left(3.75 \mathrm{~nm}^{2} / \text { molecule }\right)^{17}$ and the average surface area of the bilayer vesicles considering a diameter of $140 \mathrm{~nm}$.

Optical density measurements were carried out in $1 \mathrm{~mL}$ smallvolume disposable PMMA cuvettes at $400 \mathrm{~nm}$ by using a Uvikon 923 double-beam photospectrometer at $23{ }^{\circ} \mathrm{C}$. All solutions were prepared in HEPES buffer $\left(20 \mathrm{mM}\right.$, pH 7.45) with $1.0 \mathrm{mM} \mathrm{CaCl}_{2}$ and $\mathrm{MnCl}_{2}$. Freshly prepared vesicle solutions were used for each measurement. Each run was performed by adding the following reagents in the following order: $1 \mathrm{~mL}$ of a vesicle solution, $5 \mu \mathrm{L}$ of a carbohydrate guest stock solution, and the appropriate volume of a lectin stock solution. If the volume of the lectin solution necessary to reach the desired concentration was larger than $24 \mu \mathrm{L}$ (i.e., $2.5 \mathrm{v} / \mathrm{v} \%$ ), then the dilution of the vesicles was taken into account. The total concentration of the guest carbohydrate derivatives used was half of the concentration of CD 1. The lectin was added 3 min after the addition of the carbohydrate guest to the vesicle sample.

The change in optical density at $400 \mathrm{~nm}(\Delta \mathrm{OD} 400)$ was obtained by subtracting the initial optical density, due to the presence of carbohydrate-decorated CDV (typically <0.05), from the maximum optical density reached in the aggregation process. Control experiments were done by adding lectin to the vesicles in the absence of carbohydrate; no aggregation was observed in these cases. For each kinetic run, the initial rate was obtained from the slope of the initial linear region of the plot of optical density versus time. 
Scheme 1. Noncovalent Interactions in a Ternary System of Host Vesicles, Guest Carbohydrates, and Lectins ${ }^{a}$

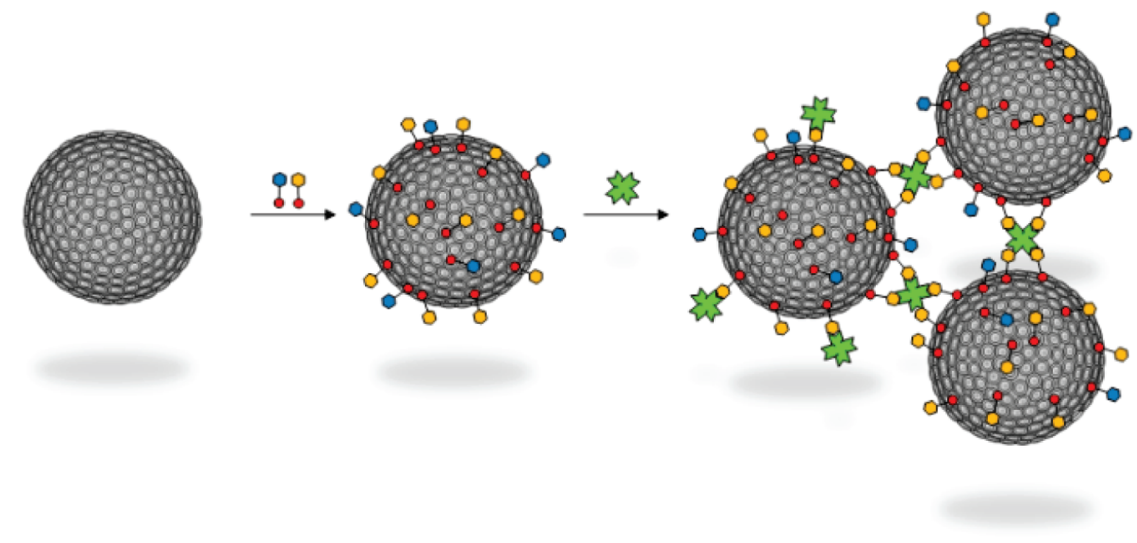

\begin{abstract}
${ }^{a}$ Lectin concanavalin A (ConA, green) induces the aggregation of cyclodextrin vesicles (CDV) in the presence of bifunctional maltose-adamantane conjugate 2 (orange). Lectin peanut agglutinin (PNA, not shown) induces the aggregation of CDV in the presence of lactose-adamantane conjugate 3 (blue).
\end{abstract}

The initial rate of aggregation was monitored to avoid complications associated with a nonlinear response from the formation of large CDV aggregates. For most concentrations, duplicate or triplicate experiments were performed to ensure the reproducibility of the data. The results shown in the Figures and Tables are the averaged values.

Dynamic light scattering measurements were performed by using a Malvern Instruments Nano-ZS instrument with smallvolume disposable PMMA cuvettes kept at $25^{\circ} \mathrm{C}$.

\section{Results and Discussion}

The aggregation in the ternary system host vesicles, guest carbohydrates, and lectins was monitored by measuring the optical density at $400 \mathrm{~nm}(\mathrm{OD} 400)$ as a function of time. The size of the vesicles as well as the aggregates formed upon addition of the lectins to the carbohydrate-decorated vesicles was measured by dynamic light scattering. The aggregation process was evaluated as a function of lectin concentration (ConA or PNA), vesicle concentration, and surface coverage of the vesicles by carbohydrates maltose $\mathbf{2}$ and lactose $\mathbf{3}$.

In the first part of this study, the aggregation of maltosedecorated CDV by ConA was investigated. To evaluate the influence of CDV concentration, the aggregation process was studied over a range of vesicle concentrations as a function of ConA concentration at $X^{\text {maltose } 2}=1.0$ (maximum surface coverage of maltose 2). The concentrations of CDV evaluated were $6.1 \times 10^{-3}$, $3.1 \times 10^{-3}, 1.5 \times 10^{-3}$, and $0.6 \times 10^{-3} \mu \mathrm{M}$, which correspond to [CD 1] equal to $200,100,50$, and $20 \mu \mathrm{M}$, respectively, and the maltose 2 concentrations used were $100,50,25$, and $10 \mu \mathrm{M}$, respectively. In view of the beta-cyclodextrin-adamantane association constant $\left(K_{\mathrm{a}} \approx 4 \times 10^{4} \mathrm{M}^{-1}\right)^{23}$ and by assuming that maltose 2 cannot permeate or very slowly permeates the vesicle membrane, it is expected that at these concentrations the carbohydrate guest is displayed at high density on the vesicle surface, whereas relatively little is present in solution. For the experiments performed with $\mathrm{CDV}$ concentrations equal to $3.1 \times 10^{-3}, 1.5 \times$ $10^{-3}$, and $0.6 \times 10^{-3} \mu \mathrm{M}$, it was possible to determine the initial rate of the aggregation process. The initial rate at $6.1 \times 10^{-3} \mu \mathrm{M}$ could not be measured accurately because of the high aggregation rate at this concentration. Figure 1 displays the correlation of the initial rates with ConA concentration at the different CDV concentrations studied. It can be seen that the initial rate of aggregation increases linearly with ConA concentration. However, the relationship between the slopes obtained from plots in Figure 1

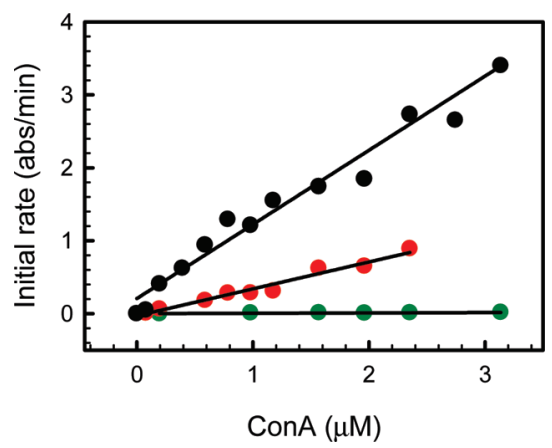

Figure 1. Kinetics of CDV aggregation by ConA. Initial rate of aggregation against ConA concentration at different $\mathrm{CDV}$ concentrations and $X^{\text {maltose } 2}=1$. $[\mathrm{CDV}]=0.6 \times 10^{-3}$ (green, $r^{2}=$ $0.72), 1.5 \times 10^{-3}\left(\mathrm{red}, r^{2}=0.97\right)$, and $3.1 \times 10^{-3}$ (black, $r^{2}=$ $0.97) \mu \mathrm{M}$.

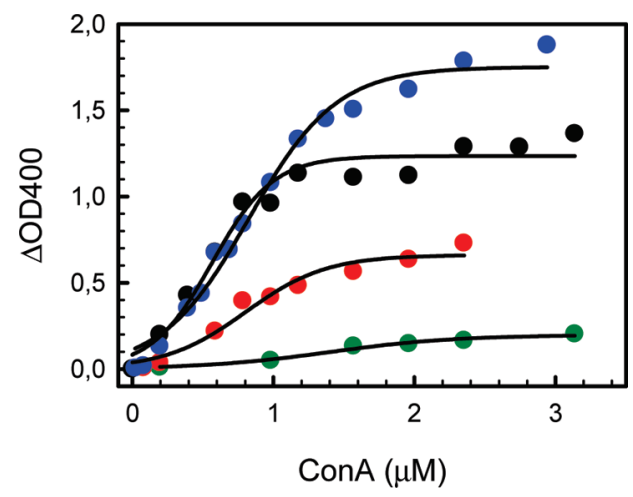

Figure 2. ConA-induced aggregation of CDV as monitored by the optical density at $400 \mathrm{~nm}$. Extent of aggregation at different CDV concentrations and $X^{\text {maltose } 2}=1$. [CDV] $=0.6 \times 10^{-3}$ (green), $1.5 \times 10^{-3}$ (red), $3.1 \times 10^{-3}$ (black), and $6.1 \times 10^{-3}$ (blue) $\mu \mathrm{M}$. The trend lines serve to guide the eye.

with CDV concentration is also linear in the range of concentrations studied, indicating that the rate of aggregation also depends linearly on the vesicle concentration (Figure S1 in the Supporting Information). The linear relationships of the initial rates of aggregation with $\mathrm{CDV}$ and ConA concentrations support the assumption that the carbohydrate guest is displayed at high density on the vesicle surface, whereas little is present in solution. 


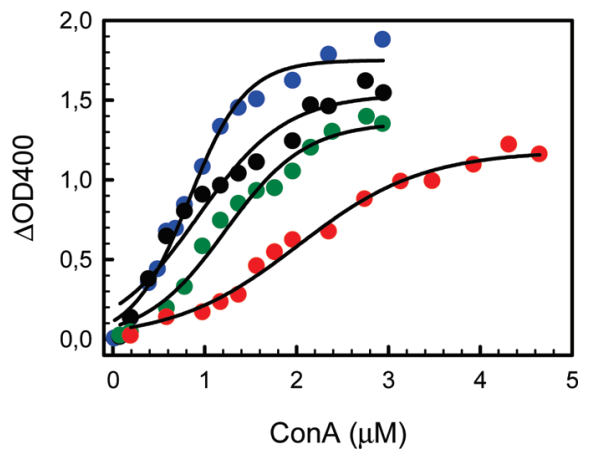

Figure 3. ConA-induced aggregation of $\mathrm{CDV}$ as monitored by the optical density at $400 \mathrm{~nm}$. Extent of aggregation of CDV at various molar fractions of maltose 2 and $[\mathrm{CDV}]=6.1 \times 10^{-3} \mu \mathrm{M}([\mathrm{CD} 1]=$ $200 \mu \mathrm{M}) . X^{\text {maltose } 2}=1.0$ (blue), 0.7 (black), 0.5 (green), and 0.3 (red). Trend lines serve to guide the eye.

Figure 2 shows the dependence of OD 400 on ConA concentration at each CDV concentration studied. It can be seen that although the extent of aggregation initially increases with ConA concentration, a maximum OD400 is reached for each CDV concentration. The trend lines in Figure 2 merely serve to guide the eye. The maximum OD400 is higher if the CDV concentration is increased, which is reasonable because a larger number of vesicles should give rise to a larger number of vesicle clusters and hence a larger OD400. The time required to reach the maximum OD400 is largest for the lower ConA and CDV concentrations (up to $120 \mathrm{~min}$ ) and decreases at higher ConA and CDV concentrations (less than $5 \mathrm{~min}$ ), consistent with the higher aggregation rates reported in Figure 1. These data are provided in Figure S2 of the Supporting Information.

Figure 3 illustrates the aggregation process as a function of ConA concentration and surface coverage of the vesicles by maltose $\mathbf{2}$. The surface coverage by maltose $\mathbf{2}$ was modified by varying the molar fraction of maltose $\mathbf{2}\left(X^{\text {maltose } \mathbf{2}}\right)$ using the lactose 3 derivative that does not bind to ConA. Because maltose $\mathbf{2}$ and lactose $\mathbf{3}$ have similar affinities for CD $\mathbf{1}^{23}$ and because the $\mathrm{CDV}$ concentration was kept constant, it can be assumed that the surface coverage of carbohydrates on the vesicle is identical to the composition of the mixture added to the vesicle solution. Figure 3 shows the results obtained at a CDV concentration of $6.1 \times 10^{-3} \mu \mathrm{M}$ (corresponding to $[\mathrm{CD} 1]=200 \mu \mathrm{M}$ ) and $[\mathbf{2}+\mathbf{3}]=$ $100 \mu \mathrm{M}$. The molar fractions of maltose 2 that were evaluated were $1.0,0.7,0.5$, and 0.3 . At each molar fraction of maltose 2 , the aggregation process was studied as a function of ConA concentration in the range of $0.2-5.0 \mu \mathrm{M}$. As can be seen in Figure 3, vesicle aggregation depends on both ConA concentration and the surface coverage of the vesicle by maltose $\mathbf{2}$. As the molar fraction of maltose $\mathbf{2}$ is gradually lowered from 1.0 to 0.3 , vesicle aggregation saturates at a lower value of $\Delta O D 400$, indicating that the average density of maltose $\mathbf{2}$ on the vesicle surface is a key factor in regulating the extent of vesicle aggregation. Similar observations were made at a lower concentration of CDV (Figure S3 in the Supporting Information).

To investigate the dependence of the aggregation process on the surface coverage of maltose $\mathbf{2}$ with respect to both the extent and the initial rate of the aggregation process, two sets of experiments were performed. First, the aggregation process was studied at a constant $\mathrm{CDV}$ concentration of $3.1 \times 10^{-3} \mu \mathrm{M}([\mathrm{CD} 1]=$ $100 \mu \mathrm{M})$ as a function of ConA concentration at 0.5 and 1.0 molar fractions of maltose 2. These results are shown in Figures S3 and S4 in the Supporting Information. Second, the aggregation process was evaluated by varying the molar fraction of maltose

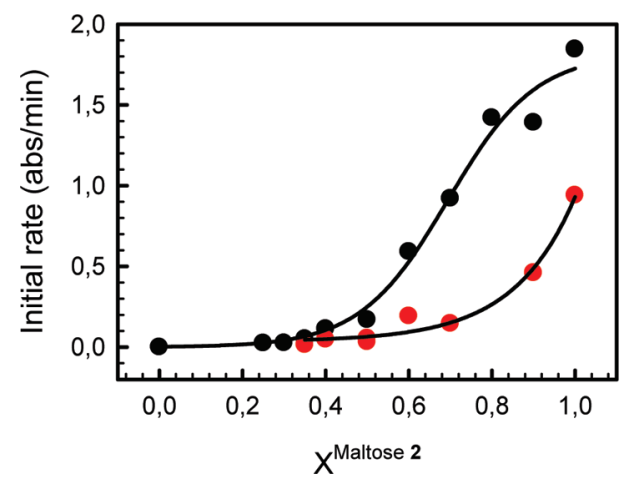

Figure 4. Kinetics of CDV aggregation by ConA. Initial rate of aggregation vs $X^{\text {maltose } 2}$ at a CDV concentration of $3.1 \times 10^{-3} \mu \mathrm{M}$ $([\mathrm{CD} 1]=100 \mu \mathrm{M})$ and $[\mathrm{ConA}]=0.59$ (red) and 1.96 (black) $\mu \mathrm{M}$. Trend lines serve to guide the eye.

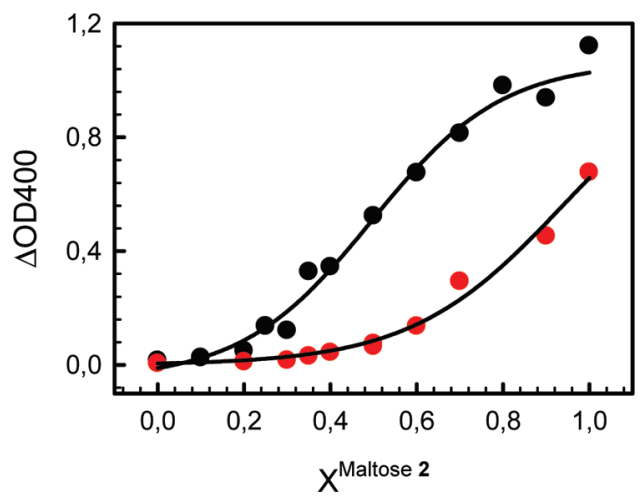

Figure 5. ConA-induced aggregation of CDV as monitored by the optical density at $400 \mathrm{~nm}$. Extent of aggregation of CDV vs $X^{\text {maltose }}$ $\mathbf{2}$ at $[\mathrm{CDV}]=3.1 \times 10^{-3} \mu \mathrm{M}([\mathrm{CD} 1]=100 \mu \mathrm{M})$ and $[\mathrm{ConA}]=$ 0.59 (red) and 1.96 (black) $\mu \mathrm{M}$. Trend lines serve to guide the eye.

2 from 0 to 1 at two ConA concentrations $(0.59$ and $1.96 \mu \mathrm{M})$ and a CDV concentration equal to $3.1 \times 10^{-3} \mu \mathrm{M}([\mathrm{CD} 1]=100 \mu \mathrm{M})$. These results are shown in Figures 4 and 5.

In Figures 2, 3, and 5, it can be seen from the $\Delta \mathrm{OD} 400$ values that as the ConA concentration is increased the aggregation process is more extensive. Also, the ConA concentration required to induce significant aggregation $(\Delta \mathrm{OD} 400>0.1)$ increases as $X^{\text {maltose } \mathbf{2}}$ decreases and vesicles with low maltose $\mathbf{2}$ coverage do not reach the same extent of aggregation even at high ConA concentration (Figures $3-5$ and S3). Furthermore, it is evident from Figures 4 and 5 that the molar fraction and hence the surface density of the carbohydrate decorating the $\mathrm{CDV}$ surface is a critical factor controlling the rate as well as the extent of vesicle aggregation.

The aggregation process depends on the establishment of orthogonal multivalent interactions between ConA and maltose $\mathbf{2}$. At low molar fractions of maltose $\mathbf{2}$, the aggregation does not reach the extent observed at high molar fractions, even at a high ratio of ConA to CDV. This indicates that a higher concentration of lectin is not enough to induce aggregation if the surface does not display a carbohydrate ligand density that is sufficient to enable the lectins to establish multivalent interactions. Upon inspection of Figures 4 and 5, it is clear that the rate of aggregation has a stronger dependence on the surface coverage of carbohydrate than the extent of aggregation. These findings suggest that there probably is a thermodynamic driving force for aggregation at a low surface density of carbohydrate but the rate becomes too low to observe a significant increase in OD400, as expected for a kinetically determined process. 
Scheme 2. Schematic Representation of CD 1 at the Vesicle Surface (Grey) with Complete and Partial Coverage by Maltose 2 (Orange ${ }^{a}$
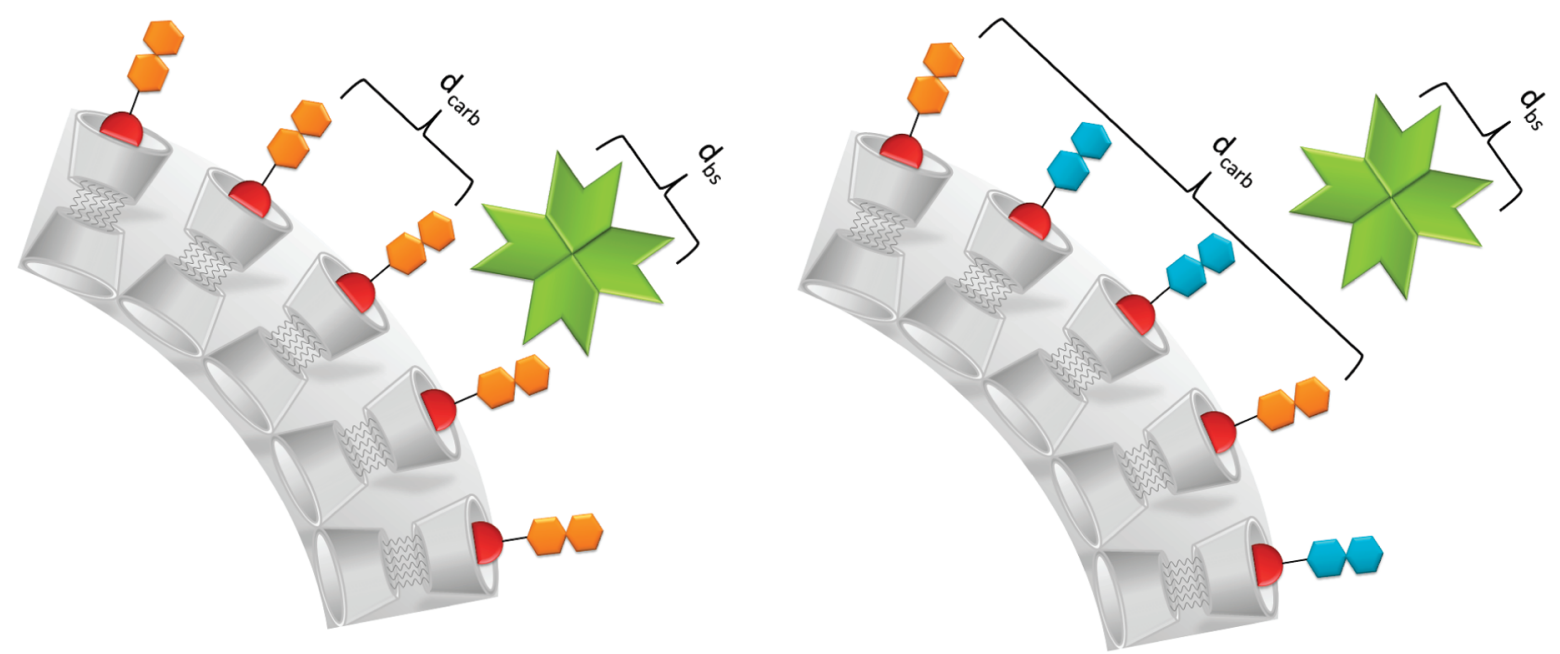

${ }^{a}$ The average separation in maltose $\mathbf{2}\left(d_{\mathrm{carb}}\right)$ is smaller (left) and larger (right) than the average binding site separation $\left(d_{\mathrm{bs}}\right)$ in ConA (green).

For aggregation to occur, the ConA tetramer must bind carbohydrates on the surfaces of two different vesicles, and this requires a two-step process in which persistent divalent binding to one vesicle is followed by binding to the second vesicle to form a stable multivalent lectin bridge. For the effective divalent binding of ConA to the vesicles, it is necessary that the maltoses are available at the surface with a distance among them equal to or smaller than the effective separation of binding sites in ConA. It was determined that the effective binding site separation for ConA binding to a glycosylated bilayer membrane is about $3.6-4.3 \mathrm{~nm} .{ }^{14}$ It should be emphasized that in our case the maximum surface density of the carbohydrate guests is limited by the spacing of the rather large $\beta$-cyclodextrin host. The average spacing of the cyclodextrins at the vesicle surface is ca. $2.2 \mathrm{~nm},{ }^{17}$ and the average spacing of carbohydrate guests at the surface at $100 \%$ coverage should be the same. A lower surface coverage of the carbohydrate that binds the lectin (and a higher surface coverage of the carbohydrate that does not bind the lectin) implies a larger average spacing of the carbohydrate ligands at the vesicle surface. Once the average spacing exceeds the distance of the carbohydrate binding sites in the lectin, multivalent binding is no longer possible and aggregation is suppressed (Scheme 2).

By assuming that in our system maltose $\mathbf{2}$ is randomly distributed on the vesicle surface and the minimal area occupied by each maltose is equal to the area occupied by CD $1\left(3.75 \mathrm{~nm}^{2}\right.$, Scheme 2), ${ }^{17}$ we estimated the average number of maltose molecules per nanometer ${ }^{2}$ (surface coverage, $\Gamma$ ) and the average separation in maltose $\left(d_{\text {carb }}\right)$ according to $\Gamma=X^{\text {maltose }} \mathbf{2} / 3.75 \mathrm{~nm}^{2}$ and $d_{\text {carb }}=2\left(3.75 \mathrm{~nm}^{2} /\left(X^{\text {maltose } 2} \pi\right)\right)^{1 / 2}$. The calculated values of $\Gamma$ and $d_{\text {carb }}$ are shown in Table $\mathrm{S} 1$ of the Supporting Information. By considering the results presented in Figures 4 and 5, it can be seen that the aggregation of CDV starts as soon as the molar fraction of maltose $\mathbf{2}$ is higher than ca. 0.30-0.35. Dynamic light scattering experiments are consistent with the OD400 measurements: when the molar fraction of maltose $\mathbf{2}$ is lower than 0.35 , the size of the carbohydrate-decorated vesicles in the presence of ConA is only slightly larger than the size of the vesicles in the absence of ConA. However, for molar fractions of maltose 2 higher than 0.35 , large polydisperse aggregates are observed. The average separation in maltose $\left(d_{\text {carb }}\right)$ is $3.4-4.0 \mathrm{~nm}$ for molar fractions between 0.30 and 0.35 (Table S1). This distance is fully consistent with the reported binding site separation in ConA. ${ }^{14}$ The experimental results show that aggregation in the

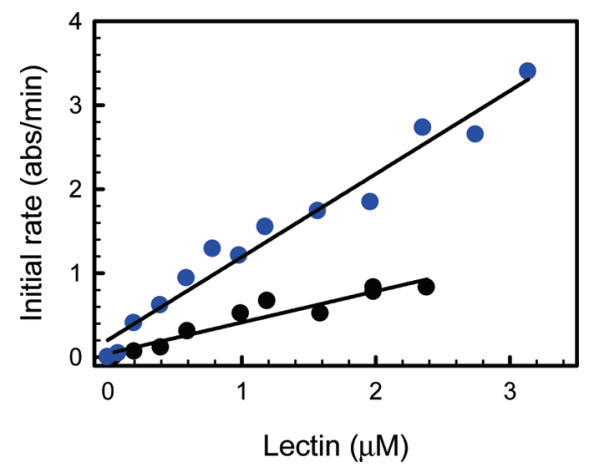

Figure 6. Kinetics of CDV aggregation. Initial rates of aggregation against lectin concentration at a CDV concentration of $3.1 \times$ $10^{-3} \mu \mathrm{M}$. ConA and $X^{\text {maltose } 2}=1$ (blue, $r^{2}=0.97$ ), and PNA and $X^{\text {lactose } 3}=1\left(\right.$ black, $\left.r^{2}=0.91\right)$.

ternary system of host vesicles, guest carbohydrates, and lectin occurs only if the average distance in maltose $2\left(d_{\text {carb }}\right)$ is equal to or smaller than the binding site separation $\left(d_{\mathrm{bs}}\right)$ in ConA (Scheme 2).

In the second part of this study, the aggregation of lactosedecorated CDV in the presence of the lectin PNA was investigated and compared to the aggregation of maltose-decorated CDV by ConA. The aggregation process was first evaluated as a function of PNA concentration by using a maximum surface coverage of lactose 3 ( $X^{\text {lactose } 3}=1.0$ ). Figures 6 and 7 provide a comparison of the initial rates and the extent of aggregation, respectively, as a function of the concentrations of PNA and ConA at [CDV] = $3.1 \times 10^{-3} \mu \mathrm{M}$ and $X^{\text {tactose } 3}=1$ (in the case of PNA) or $X^{\text {maltose } 2}=1$ (in the case of ConA). Also, the effect on the variation of $X^{\text {lactose } 3}$ between 0 and 1 was investigated by using a constant concentration of $[\mathrm{CDV}]=3.1 \times 10^{-3} \mu \mathrm{M}$ and $[\mathrm{PNA}]=1.98 \mu \mathrm{M}$ or $[\mathrm{ConA}]=$ $1.96 \mu \mathrm{M}$. Figures 8 and 9 compare the initial rates and the extent of aggregation, respectively, at a constant concentration of each lectin and different molar fractions of the carbohydrates.

The results obtained with PNA show trends that are similar to those observed for ConA. When comparing the initial rate of the aggregation process (Figure 6), it is observed that the ConAinduced aggregation of CDV is significantly faster than the PNAinduced aggregation of CDV. The slopes in Figure 6 show that aggregation by Con $\mathrm{A}$ is almost 3 times faster than aggregation by PNA. As can be seen in Figure 7, the extent of aggregation is 


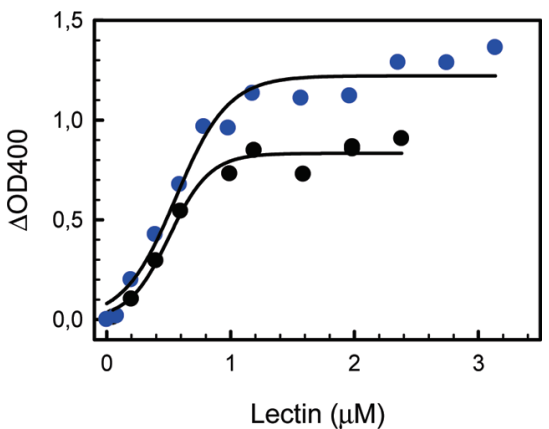

Figure 7. Lectin-induced aggregation of CDV as monitored by the optical density at $400 \mathrm{~nm}$. Extent of lectin-induced aggregation at a molar fraction $X$ of maltose $\mathbf{2}$ or lactose $\mathbf{3}$ equal to 1 and [CDV] $=$ $3.1 \times 10^{-3} \mu \mathrm{M}([\mathrm{CD} 1]=100 \mu \mathrm{M})$. ConA (blue) and PNA (black). Trend lines serve to guide the eye.

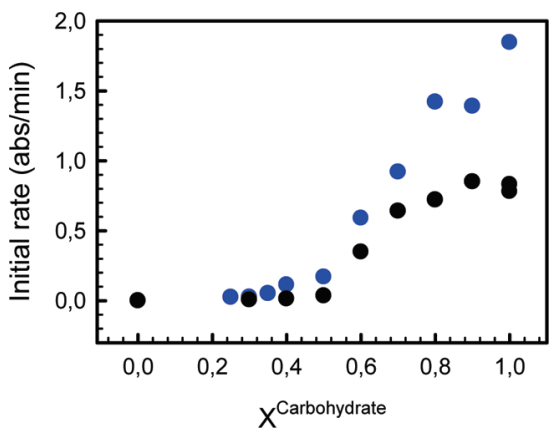

Figure 8. Kinetics of CDV aggregation. Initial rate of aggregation vs the molar fraction of maltose $\mathbf{2}$ (blue) or lactose $\mathbf{3}$ (black) at $[\mathrm{CDV}]=3.1 \times 10^{-3} \mu \mathrm{M}([\mathrm{CD} \mathrm{1}]=100 \mu \mathrm{M})$ and $[\mathrm{ConA}]=$ 1.96 (blue) or [PNA] $=1.98$ (black) $\mu \mathrm{M}$.

nearly identical for both lectins, with a slight difference at high lectin concentrations $(>1 \mu \mathrm{M})$. Upon inspection of the dependence of the aggregation process on the molar fraction of carbohydrate on the vesicle surface (Figures 8 and 9), it can be seen that, within the concentration range investigated here, both lectins exhibit similar behavior, with the difference being that PNA induces the aggregation of CDV at slightly higher molar fractions of the carbohydrate. The PNA-induced aggregation of lactosedecorated vesicles requires a molar fraction of lactose $\mathbf{3}$ that is higher than 0.4 to 0.5 . This finding indicates that the separation of the binding sites of PNA is significantly smaller than that of ConA. The average binding site separation estimated for PNA is about 2.4 to $3.0 \mathrm{~nm}$ (Table S1). To the best of our knowledge, there is no comparable measurement of the effective binding site separation of PNA. We note that the effective binding site separation cannot be accurately determined from the crystal structure of the lectin. ${ }^{24,25}$

The equilibrium binding constants $K_{\mathrm{a}}$ determined for the formation of a 1:1 complex between ConA and maltose ${ }^{26}$ and between PNA and lactose, ${ }^{27}$ respectively, are both $1.3 \times 10^{3} \mathrm{M}^{-1}$. Also, the association rate constants $k_{\mathrm{a}}$ determined for the formation of a 1:1 complex between ConA and methyl- $\beta$-glucosides

(24) Bouckaert, J.; Hamelryck, T. W.; Wyns, L.; Loris, R. J. Biol. Chem. 1999 274, 29188-29195.

(25) Banerjee, R.; Das, K.; Ravishankar, R.; Suguna, K.; Surolina, A.; Vijayan, M. J. Mol. Biol. 1996, 259, 281-296.

(26) (a) Mandal, D. K.; Kishore, N.; Brewer, C. F. Biochemistry 1994, 33, 1149 1156. (b) Dam, T. K.; Oscarson, S.; Sacchettini, J. C.; Brewer, C. F. J. Biol. Chem. 1998, $273,32826-32832$.

(27) Neurohr, K. J.; Young, N. M.; Mantsch, H. H. J. Biol. Chem. 1980, 255, 9205-9209.

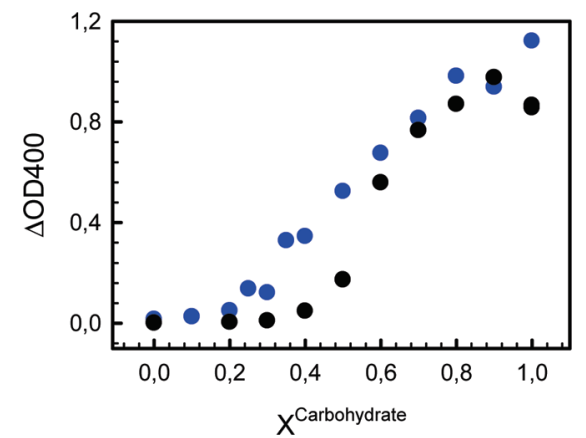

Figure 9. Lectin-induced aggregation of CDV as monitored by the optical density at $400 \mathrm{~nm}$. Extent of lectin-induced aggregation at different molar fractions of maltose $\mathbf{2}$ (blue) or lactose $\mathbf{3}$ (black) at $[\mathrm{CDV}]=3.1 \times 10^{-3} \mu \mathrm{M}([\mathrm{CD} 1]=100 \mu \mathrm{M})$ and $\mathrm{ConA}=$ 1.96 (blue) or PNA $=1.98$ (black) $\mu \mathrm{M}$.

$\left(5.0 \times 10^{4} \mathrm{M}^{-1} \mathrm{~s}^{-1}\right)^{28}$ and between PNA and methyl- $\alpha$-galactosides $\left(3.7 \times 10^{4} \mathrm{M}^{-1} \mathrm{~s}^{-1}\right)^{29}$ are known. Although the formation of the cyclodextrin-adamantane complex is essentially diffusioncontrolled, the association rates of the carbohydrate-lectin complexes are thus several orders of magnitude smaller. However, the remarkable difference in the initial rate of aggregation in the ternary system can hardly be explained by the slight difference in the association rate of the monovalent carbohydrate-lectin complexes. The rate of vesicle aggregation should be strongly dependent on the number of carbohydrate ligands accessible to the lectin binding pockets and on the separation and orientation of the carbohydrates on the vesicle surface. Two critical factors could potentially explain the different rates of ConA- and PNAinduced aggregation: (1) the association rate $k_{\mathrm{a}}$ of the carbohydrates with the lectins on the vesicle surface and (2) the clustering of carbohydrate ligands on the vesicle surface.

It is well established that a multivalent arrangement of carbohydrate ligands on a surface (such as a vesicle surface, a selfassembled monolayer, or a polymer) results in a significantly higher association rate $k_{\mathrm{a}}$ and a much lower dissociation rate $k_{\mathrm{d}}{ }^{30}$ However, it has also been reported that the kinetics of multivalent carbohydrate-lectin interactions vary with the carbohydrate, the carbohydrate surface density, and the lectin, and these parameters provide a unique kinetic response for each carbohydrate-lectin pair. ${ }^{31}$ According to our results, ConA interacts significantly more rapidly with maltose $\mathbf{2}$ than PNA interacts with lactose $\mathbf{3}$, although both carbohydrates are presented in identical fashion on the CDV surface.

It could be argued that the higher aggregation rate of ConA is caused by clustering of the carbohydrate ligands, possibly induced or enhanced by the multivalent receptor. In this scenario, the mixed glycocalix of maltose $\mathbf{2}$ and lactose $\mathbf{3}$ is not entirely homogeneous but instead contains patches that are rich in maltose $\mathbf{2}$ and other patches that are rich in lactose $\mathbf{3}$. In that case, it would be expected that ConA (with a larger effective binding site separation) would induce faster aggregation because it can bind two maltose molecules at a larger separation, whereas PNA (with a smaller effective binding site separation) would induce slower aggregation because it can bind only two lactose molecules

(28) Brewer, C. F.; Marcus, D.; Grollman, A. P.; Sternlicht, H. In Lysozyme; Osserman, A., Beychok, S., Canfield, R., Eds.; Academic Press: New York, 1972; pp 239-250.

(29) Neurohr, K. J.; Young, N. M.; Smith, I. C. P.; Mantsch, H. H. Biochemistry 1981, 20, 3499-3504

(30) (a) Kiessling, L. N.; Pohl, N. L. Chem. Biol. 1996, 3, 71-77. (b) Mammen, M.; Choi, S. K.; Whitesides, G. M. Angew. Chem., Int. Ed. 1998, 37, 2754-2794.

(31) Fukuda, T.; Onogi, S.; Miura, Y. Thin Solid Films 2009, 518, 880-888. 
in close proximity. However, it is difficult to explain why very similar molecules such as maltose $\mathbf{2}$ and lactose $\mathbf{3}$ would not distribute completely homogeneously on the vesicle surface. Therefore, we propose that the mixed glycocalix is homogeneous in the absence of lectin but clusters of maltose $\mathbf{2}$ are formed in the presence of ConA (and clusters of lactose $\mathbf{3}$ are formed in the presence of PNA) in order to maximize the interaction with the multivalent lectin. Also in this case it would be expected that ConA (with a larger effective binding site separation) would induce a faster aggregation than PNA (with a smaller effective binding site separation). This type of receptor-induced ligand clustering is conceptually identical to ligand-induced receptor clustering known from biological membranes, ${ }^{2}$ and we have also invoked this dynamic phenomenon in previous investigations of cyclodextrin vesicles. $^{22}$

\section{Conclusions}

In this article, we demonstrate that a self-assembled glycocalix at the surface of cyclodextrin vesicles is a dynamic system in which several orthogonal noncovalent interactions operate simultaneously. In addition, the self-assembled glycocalix serves as a platform on which to study the multivalent interaction between carbohydrates and lectins at interfaces. It was found that the initial rate of aggregation scales linearly with the lectin as well as the vesicle concentration and that each lectin requires a characteristic critical density of complementary carbohydrates at the vesicle surface. These observations allow us to predict the response of the ternary supramolecular system at different concentrations and stoichiometry of its components. This methodology can be extended to the study of interactions in other ligand-receptor systems at interfaces because of the straightforward control of the ligand surface density: the composition of the glycocalix can be adjusted simply by changing the ratio of guest molecules. Also, in a simple manner this model system permits us to estimate the binding site distance in a multivalent receptor such as a multiple binding site protein. Ultimately, these results will further our understanding of molecular recognition at biological interfaces as well as the self-assembly of dynamic soft materials.

Acknowledgment. This work was supported by the Deutsche Forschungsgemeinschaft (DFG grant Ra 1732/1-1). R.V.V. is a grateful recipient of a fellowship from the Deutscher Akademischer Austauschdienst (DAAD).

Supporting Information Available: Additional plots of the initial rate and $\triangle O D 400$ as a function of vesicle concentration, lectin concentration, and molar fraction of carbohydrate. Average density and separation of carbohydrate ligands on the vesicle surface. Values of $\Delta O D 400$ and initial rates of the plots presented in the text and Supporting Information. This material is available free of charge via the Internet at http://pubs.acs.org. 